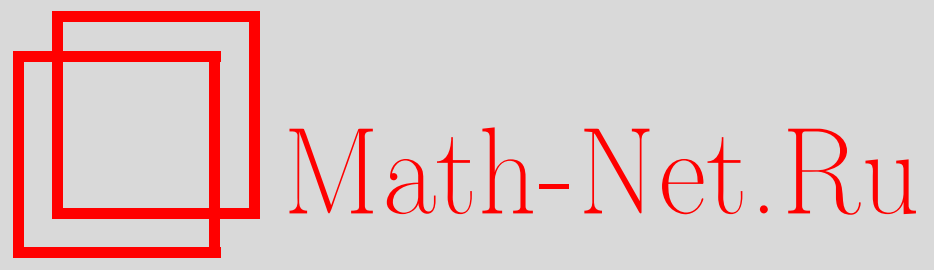

А. М. Ланге, О распределении числа финальных частиц ветвящегося процесса с превращениями и парными взаимодействиями, Теория вероятн. и ее примен., 2006, том 51, выпуск 4, 801-809

DOI: https://doi.org/10.4213/tvp28

Использование Общероссийского математического портала Math-Net.Ru подразумевает, что вы прочитали и согласны с пользовательским соглашением

http://www . mathnet.ru/rus/agreement

Параметры загрузки:

IP : 54.198 .187 .58

26 апреля 2023 г., 11:13:05 
3. Malyshev V. A. Microscopic models for chemical thermodynamics. - J. Statist. Phys., 2005 , v. 119 , № 5/6, p. 997-1026.

4. Malyshev V. A. Fixed points for stochastic open chemical systems. - Markov Process. Related Fields, 2005, v. 11, № 2, p. 337-354.

5. Gadgil C., Lee C.-H., Othmer H.G. A stochastic analysis of first-order reaction networks. - Bull. Math. Biol., 2005, v. 67, № 5, p. 901-946.

6. Streater R. F. Statistical Dynamics. London: Imperial College Press, 1995, 275 p.

7. Thomas $R$. Feedback loops: the wheels of regulatory networks. - Integrative Approaches to Molecular Biology. Ed. by J. Collado-Vides, B. Magasanik, and T.F. Smith. Cambridge: MIT Press, 1996, p. 167-178.

8. Mavrovouniotis M.L. Analysis of complex metabolic pathways. - Integrative Approaches to Molecular Biology. Ed. by J. Collado-Vides, B. Magasanik, and T.F. Smith. Cambridge: MIT Press, 1996, p. 211-238.

9. Fell D. Understanding the Control of Metabolism. London: Portland Press, 1996, $300 \mathrm{p}$.

10. Левитан Б. М. Почти-периодические функции. М.: Гостехиздат, 1953, 396 с.

11. Rybko A., Shlosman S. Poisson hypothesis for information networks. I, II. - Moscow Math. J., 2005, v. 5, № 3, p. 679-704; № 4, p. 927-959.

12. Rybko A., Shlosman S., Vladimirov A. Self-averaging property of queueing systems. http://www.arxiv.org: math.PR/0510046.

13. Rolski T. Ergodic properties of Poisson processes with almost periodic intensity. Probab. Theory Related Fields, 1990, v. 84, № 1, p. 27-37.

Поступила в редакцию

21.XII. 2005

(C) 2006 г.

ЛАНГЕ А. M.*

\title{
О РАСПРЕДЕЛЕНИИ ЧИСЛА ФИНАЛЬНЫХ ЧАСТИЦ ВЕТВЯЩЕГОСЯ ПРОЩЕССА С ПРЕВРАЩЕНИЯМИ И ПАРНЫМИ ВЗАИМОДЕЙСТВИЯМИ
}

\begin{abstract}
Рассматривается марковский ветвящийся процесс с непрерывным временем и двумя типами частиц $T_{1}$ и $T_{2}$. Частицы обоих типов образуются при превращении частицы типа $T_{1}$ или взаимодействии двух частиц типа $T_{1}$. При частных предположениях о распределениях числа новых частиц найдены асимптотики математического ожидания и дисперсии и показана асимптотическая нормальность распределения числа финальных частиц типа $T_{2}$ при большом начальном числе частиц типа $T_{1}$.
\end{abstract}

Ключевые слова и фразы:: ветвящийся процесс с взаимодействием, финальные вероятности, экспоненциальная производящая функция, стационарное первое уравнение Колмогорова, точные решения.

1. Ветвящийся процесс с двумя типами частиц $T_{1}, T_{2}$ и двумя комплексами взаимодействия $\varepsilon^{1}=(1,0), \varepsilon^{2}=(2,0)[\mathbf{5}]$. Пусть $\xi(t), t \in[0, \infty)$, 一 однородный во времени марковский процесс на множестве состояний $\mathbf{N}^{2}=\left\{\left(\alpha_{1}, \alpha_{2}\right), \alpha_{1}, \alpha_{2}=\right.$ $0,1,2, \ldots\}$. Переходные вероятности $P_{\left(\beta_{1}, \beta_{2}\right)}^{\left(\alpha_{1}, \alpha_{2}\right)}(t)=\mathbf{P}\left(\xi(t)=\left(\beta_{1}, \beta_{2}\right) \mid \xi(0)=\left(\alpha_{1}, \alpha_{2}\right)\right)$ при $\Delta t \rightarrow 0$ имеют вид $\left(\lambda_{1}>0, \lambda_{2}>0\right)$

$$
\begin{aligned}
& P_{\left(\alpha_{1}, \alpha_{2}\right)}^{\left(\alpha_{1}, \alpha_{2}\right)}(\Delta t)=1-\left(\lambda_{2} \alpha_{1}\left(\alpha_{1}-1\right)+\lambda_{1} \alpha_{1}\right) \Delta t+o(\Delta t), \\
& P_{\left(\beta_{1}, \beta_{2}\right)}^{\left(\alpha_{1}, \alpha_{2}\right)}(\Delta t)=\left(\lambda_{2} \alpha_{1}\left(\alpha_{1}-1\right) p_{\beta_{1}-\alpha_{1}+2, \beta_{2}-\alpha_{2}}^{2}+\lambda_{1} \alpha_{1} p_{\beta_{1}-\alpha_{1}+1, \beta_{2}-\alpha_{2}}^{1}\right) \Delta t+o(\Delta t),
\end{aligned}
$$

* Московский государственный технический университет им. Н.Э. Баумана, 2-я Бауманская ул., 5, 107005 Москва, Россия; e-mail: lange_am@hotmail.com 
если $\alpha_{1} \neq \beta_{1}$ или $\alpha_{2} \neq \beta_{2}$. Здесь $\left\{p_{\gamma_{1} \gamma_{2}}^{l} \geqslant 0, \gamma_{1}, \gamma_{2} \in \mathbf{N} ; \sum_{\gamma_{1} \gamma_{2}=0}^{\infty} p_{\gamma_{1} \gamma_{2}}^{l}=1, p_{l 0}^{l}=0\right\}$, $l=1,2,-$ заданные распределения вероятностей. Введем производящие функции

$$
\begin{aligned}
G_{\left(\beta_{1}, \beta_{2}\right)}\left(t ; z_{1}, z_{2}\right) & =\sum_{\alpha_{1}, \alpha_{2}=0}^{\infty} \frac{z_{1}^{\alpha_{1}} z_{2}^{\alpha_{2}}}{\alpha_{1} ! \alpha_{2} !} P_{\left(\beta_{1}, \beta_{2}\right)}^{\left(\alpha_{1}, \alpha_{2}\right)}(t), \\
F_{\left(\alpha_{1}, \alpha_{2}\right)}\left(t ; s_{1}, s_{2}\right) & =\sum_{\beta_{1}, \beta_{2}=0}^{\infty} P_{\left(\beta_{1}, \beta_{2}\right)}^{\left(\alpha_{1}, \alpha_{2}\right)}(t) s_{1}^{\beta_{1}} s_{2}^{\beta_{2}}, \\
h_{l}\left(s_{1}, s_{2}\right) & =\sum_{\gamma_{1}, \gamma_{2}=0}^{\infty} p_{\gamma_{1} \gamma_{2}}^{l} s_{1}^{\gamma_{1}} s_{2}^{\gamma_{2}}, \quad\left|s_{1}\right| \leqslant 1, \quad\left|s_{2}\right| \leqslant 1,
\end{aligned}
$$

и линейные дифференциальные операторы

$$
h_{l}\left(\frac{\partial}{\partial z_{1}}, \frac{\partial}{\partial z_{2}}\right)=\sum_{\gamma_{1}, \gamma_{2}=0}^{\infty} p_{\gamma_{1} \gamma_{2}}^{l} \frac{\partial^{\gamma_{1}+\gamma_{2}}}{\partial z_{1}^{\gamma_{1}} \partial z_{2}^{\gamma_{2}}}, \quad l=1,2 .
$$

Первая (обратная) система дифференциальных уравнений Колмогорова для переходных вероятностей марковского процесса $\xi(t)$ равносильна уравнению в частных производных (см. [5, теорема 2])

$$
\frac{\partial G_{\left(\beta_{1}, \beta_{2}\right)}}{\partial t}=\left[\lambda_{2} z_{1}^{2}\left(h_{2}\left(\frac{\partial}{\partial z_{1}}, \frac{\partial}{\partial z_{2}}\right)-\frac{\partial^{2}}{\partial z_{1}^{2}}\right)+\lambda_{1} z_{1}\left(h_{1}\left(\frac{\partial}{\partial z_{1}}, \frac{\partial}{\partial z_{2}}\right)-\frac{\partial}{\partial z_{1}}\right)\right] G_{\left(\beta_{1}, \beta_{2}\right)}
$$

с начальным условием $G_{\left(\beta_{1}, \beta_{2}\right)}\left(0 ; z_{1}, z_{2}\right)=z_{1}^{\beta_{1}} z_{2}^{\beta_{2}} /\left(\beta_{1} ! \beta_{2} !\right)$.

Вторая (прямая) система дифференциальных уравнений Колмогорова равносильна уравнению в частных производных (см. [5, теорема 1])

$$
\frac{\partial F_{\left(\alpha_{1}, \alpha_{2}\right)}}{\partial t}=\lambda_{2}\left(h_{2}\left(s_{1}, s_{2}\right)-s_{1}^{2}\right) \frac{\partial^{2} F_{\left(\alpha_{1}, \alpha_{2}\right)}}{\partial s_{1}^{2}}+\lambda_{1}\left(h_{1}\left(s_{1}, s_{2}\right)-s_{1}\right) \frac{\partial F_{\left(\alpha_{1}, \alpha_{2}\right)}}{\partial s_{1}}
$$

с начальным условием $F_{\left(\alpha_{1}, \alpha_{2}\right)}\left(0 ; s_{1}, s_{2}\right)=s_{1}^{\alpha_{1}} s_{2}^{\alpha_{2}}$.

Состояние $\left(\alpha_{1}, \alpha_{2}\right)$ интерпретируется как наличие $\alpha_{1}$ частиц типа $T_{1}$ и $\alpha_{2}$ частиц типа $T_{2}[1],[2],[5]$. Через случайное время $\tau_{\left(\alpha_{1}, \alpha_{2}\right)}^{1}, \mathbf{P}\left\{\tau_{\left(\alpha_{1}, \alpha_{2}\right)}^{1}<t\right\}=1-\exp \left\{-\lambda_{1} \alpha_{1} t\right\}$, частица типа $T_{1}$ независимо от других частиц может превратиться в совокупность из $\gamma_{1}$ частиц типа $T_{1}$ и $\gamma_{2}$ частиц типа $T_{2}$ с распределением вероятностей $\left\{p_{\gamma_{1} \gamma_{2}}^{1}\right\}$. Кроме того, через случайное время $\tau_{\left(\alpha_{1}, \alpha_{2}\right)}^{2}, \mathbf{P}\left\{\tau_{\left(\alpha_{1}, \alpha_{2}\right)}^{2}<t\right\}=1-\exp \left\{-\lambda_{2} \alpha_{1}\left(\alpha_{1}-1\right) t\right\}$, пара частиц $T_{1}+T_{1}$ независимо от других частиц может превратиться в группу частиц с распределением вероятностей $\left\{p_{\gamma_{1} \gamma_{2}}^{2}\right\}$. Предполагается, что случайные величины $\tau_{\left(\alpha_{1}, \alpha_{2}\right)}^{1}$ и $\tau_{\left(\alpha_{1}, \alpha_{2}\right)}^{2}$ независимы. В состоянии $\left(\alpha_{1}, \alpha_{2}\right)$ процесс находится случайное время $\tau_{\left(\alpha_{1}, \alpha_{2}\right)}=\min \left(\tau_{\left(\alpha_{1}, \alpha_{2}\right)}^{1}, \tau_{\left(\alpha_{1}, \alpha_{2}\right)}^{2}\right)$. Частицы типа $T_{2}$ называются финальными, их число не уменьшается со временем и не влияет на число частиц типа $T_{1}$ [4].

2. Задача о финальных вероятностях. Для марковского процесса $\xi(t)$ состояния $\left(0, \beta_{2}\right), \beta_{2} \in \mathbf{N}$, являются поглощающими; пусть начальное состояние $\xi(0)=\left(\alpha_{1}, 0\right), \alpha_{1} \in \mathbf{N}$. Введем финальные вероятности $q_{\left(0, \beta_{2}\right)}^{\left(\alpha_{1}, 0\right)}=\lim _{t \rightarrow \infty} P_{\left(0, \beta_{2}\right)}^{\left(\alpha_{1}, 0\right)}(t)$ и вероятность вырождения $q_{\alpha_{1} 0}=\sum_{\beta_{2}=0}^{\infty} q_{\left(0, \beta_{2}\right)}^{\left(\alpha_{1}, 0\right)}$. Обозначим $\eta_{\alpha_{1}}$ случайное число частиц типа $T_{2}$, оставшихся после вырождения процесса. Финальное распределение $\left\{q_{\left(0, \beta_{2}\right)}^{\left(\alpha_{1}, 0\right)}, \beta_{2} \in \mathbf{N}\right\}$ может быть несобственным, т.е. $q_{\alpha_{1} 0} \leqslant 1$.

Введем производяшие функции

$$
f_{\alpha_{1}}(u)=\sum_{\beta_{2}=0}^{\infty} q_{\left(0, \beta_{2}\right)}^{\left(\alpha_{1}, 0\right)} u^{\beta_{2}}, \quad w_{0}(z, u)=\sum_{\alpha_{1}=0}^{\infty} \frac{z^{\alpha_{1}}}{\alpha_{1} !} f_{\alpha_{1}}(u), \quad|u| \leqslant 1 .
$$

Положим $h_{\gamma_{1}}^{l}(u)=\sum_{\gamma_{2}=0}^{\infty} p_{\gamma_{1} \gamma_{2}}^{l} u^{\gamma_{2}},|u| \leqslant 1 ;$ пусть $p_{\gamma_{1}}^{l}=\sum_{\gamma_{2}=0}^{\infty} p_{\gamma_{1} \gamma_{2}}^{l}$, $l=1,2$. Параметры критичности ветвяшегося процесса обозначим $a_{l}=$ $\lambda_{l}\left(\left.\left(\partial h_{l}(s, u) / \partial s\right)\right|_{s=1, u=1}-l\right), l=1,2[4]$. 
В случае $\lambda_{1}>0, \lambda_{2}=0$ (ветвящийся процесс с независимыми частицами) известно, что $q_{\alpha_{1} 0}=q_{1}^{\alpha_{1}}$, где $q_{1}$ - ближайший к нулю неотрицательный корень уравнения $h_{1}(s, 1)-s=0[4$, гл. $2, \S 1]$. В докритическом $\left(a_{1}<0\right)$ и надкритическом $\left(a_{1}>0\right)$ процессах финальное распределение асимптотически нормально при $\alpha_{1} \rightarrow \infty$, а в критическом $\left(a_{1}=0\right)$ процессе финальное распределение при $\alpha_{1} \rightarrow \infty$ отлично от нормального закона $[4$, гл. $5, \S 5]$.

В случае $\lambda_{1}=0, \lambda_{2}>0$ в работе [5] найдена асимптотика $q_{\alpha_{1} 0} \sim C_{0} q_{2}^{\alpha_{1}}$, $\alpha_{1} \rightarrow \infty$, где $C_{0}>0$ и $q_{2}$ - ближайший к нулю неотрицательный корень уравнения $h_{2}(s, 1)-s^{2}=0$. В [6] получены предельные теоремы для финальных распределений в докритическом $\left(a_{2}<0\right)$, критическом $\left(a_{2}=0\right)$ и надкритическом $\left(a_{2}>0\right)$ процессах, аналогичные результатам [4].

В случае $\lambda_{1}>0, \lambda_{2}>0$ для надкритического ( $\left.a_{2}>0\right)$ процесса при $h_{1}(s, u)=$ $h_{0}^{1}(u)+h_{2}^{1}(u) s^{2}, h_{2}(s, u)=h_{0}^{2}(u)+h_{1}^{2}(u) s+h_{3}^{2}(u) s^{3}$ и условиях $p_{2}^{1}>0, p_{3}^{2}>0, p_{0}^{1}+p_{0}^{2}>0$, $p_{0}^{2}+p_{1}^{2}>0, q_{2}>q_{1}$ найдена асимптотика $q_{\alpha_{1} 0} \sim C q_{2}^{\alpha_{1}} / \alpha_{1}^{\alpha}, \alpha_{1} \rightarrow \infty$, где $C>0$ и $\alpha>0$ $[7$, следствие 1$]$.

В настоящей работе рассматривается докритический $\left(a_{2}<0\right)$ процесс. Выражения для производящей функции $f_{\alpha_{1}}(u)$, асимптотические оценки для математического ожидания $m_{\alpha_{1}}=\mathbf{E} \eta_{\alpha_{1}}$, дисперсии $\sigma_{\alpha_{1}}^{2}=\mathbf{D} \eta_{\alpha_{1}}$ при $\alpha_{1} \rightarrow \infty$ и асимптотическая нормальность финального распределения установлены при дополнительных предположениях о распределениях вероятностей $\left\{p_{\gamma_{1} \gamma_{2}}^{l}\right\}, l=1,2$. Производящая функция $w_{0}(z, u)$ находится как точное решение стационарного уравнения Колмогорова.

Предельные распределения ветвящихся процессов с парными взаимодействиями рассматривались в работах [11], [12].

3. Первое уравнение Колмогорова для экспоненциальной производяшей функции финальных вероятностей. Введем производящую функцию [6]

$$
W_{\beta_{1}}(t ; z, u)=\sum_{\alpha_{1}=0, \beta_{2}=0}^{\infty} \frac{z^{\alpha_{1}}}{\alpha_{1} !} P_{\left(\beta_{1}, \beta_{2}\right)}^{\left(\alpha_{1}, 0\right)}(t) u^{\beta_{2}}, \quad \beta_{1} \in \mathbf{N}
$$

и дифференциальные операторы

$$
h_{l}\left(\frac{\partial}{\partial z_{1}}, u\right)=\sum_{\gamma_{1}, \gamma_{2}=0}^{\infty} p_{\gamma_{1} \gamma_{2}}^{l} u^{\gamma_{2}} \frac{\partial^{\gamma_{1}}}{\partial z_{1}^{\gamma_{1}}}, \quad|u| \leqslant 1, \quad l=1,2 .
$$

Теорема 1. Производящая Функиия переходных вероятностей $W_{\beta_{1}}(t ; z, u) n p u$ любом $\beta_{1} \in \mathbf{N}$ удовлетворяет линейному дифференциальному уравнению в частных производных

$$
\frac{\partial W_{\beta_{1}}}{\partial t}=\left[\lambda_{2} z^{2}\left(h_{2}\left(\frac{\partial}{\partial z}, u\right)-\frac{\partial^{2}}{\partial z^{2}}\right)+\lambda_{1} z\left(h_{1}\left(\frac{\partial}{\partial z}, u\right)-\frac{\partial}{\partial z}\right)\right] W_{\beta_{1}}
$$

с начальньм условием $W_{\beta_{1}}(0 ; z, u)=z^{\beta_{1}} / \beta_{1}$ !.

Вывод уравнения (3) основан на уравнении (1) и соотношениях для переходных вероятностей $P_{\left(\beta_{1}, \beta_{2}\right)}^{\left(\alpha_{1}, \alpha_{2}\right)}(t)=P_{\left(\beta_{1}, \beta_{2}-\alpha_{2}\right)}^{\left(\alpha_{1}, 0\right)}(t)$ при $\alpha_{2} \leqslant \beta_{2}, P_{\left(\beta_{1}, \beta_{2}\right)}^{\left(\alpha_{1}, \alpha_{2}\right)}(t)=0$ при $\alpha_{2}>\beta_{2}$ (ср. $[6$, теорема 1]).

Аналогично работе [6] показывается, что $w_{0}(z, u)=\lim _{t \rightarrow \infty} W_{0}(t ; z, u)$ и функция $w_{0}(z, u)$ удовлетворяет стационарному первому уравнению

$$
\left[\lambda_{2} z\left(h_{2}\left(\frac{d}{d z}, u\right)-\frac{d^{2}}{d z^{2}}\right)+\lambda_{1}\left(h_{1}\left(\frac{d}{d z}, u\right)-\frac{d}{d z}\right)\right] w_{0}(z, u)=0
$$

Из равенства $q_{(0,0)}^{(0,0)}=1$ следует условие $w_{0}(0, u) \equiv 1$. Функция $w_{0}(z, u)$ при $|u|<1$ является аналитической функцией переменных $z$ и $u$, так как

$$
\left|w_{0}(z, u)\right| \leqslant \sum_{\alpha_{1}=0}^{\infty} \frac{|z|^{\alpha_{1}}}{\alpha_{1} !} \sum_{\beta_{2}=0}^{\infty} q_{\left(0, \beta_{2}\right)}^{\left(\alpha_{1}, 0\right)}|u|^{\beta_{2}} \leqslant \sum_{\alpha_{1}=0}^{\infty} \frac{|z|^{\alpha_{1}}}{\alpha_{1} !}=e^{|z|} .
$$


Обыкновенное дифференциальное уравнение (4), коэффициенты которого являются линейными функциями переменной $z$, относится к типу уравнений Лапласа $[10$, ч. 1 , гл. $5, \S 22.4]$. Решение строится методом определенного интеграла

$$
w_{0}(z, u)=\int_{K} e^{z s} \varphi(s, u) d s
$$

где

$$
\varphi(s, u)=\frac{1}{h_{2}(s, u)-s^{2}} \exp \left(\frac{\lambda_{1}}{\lambda_{2}} \int \frac{h_{1}(v, u)-v}{h_{2}(v, u)-v^{2}} d v\right),
$$

причем кривая $K$ в комплексной $s$-плоскости удовлетворяет условию

$$
\int_{K} \frac{d}{d s} e^{z s}\left(h_{2}(s, u)-s^{2}\right) \varphi(s, u) d s=0 .
$$

Нахождение кривой $K$ связано с рассмотрением римановой поверхности, соответствующей функции $\varphi(s, u)[10]$.

4. Интегральное представление для производящей функции $f_{\alpha_{1}}(u)$. Случай $h_{1}(s, u)=h_{0}^{1}(u)+h_{1}^{1}(u) s+h_{2}^{1}(u) s^{2}, h_{2}(s, u)=h_{0}^{2}(u)+h_{1}^{2}(u) s+h_{2}^{2}(u) s^{2}$, $p_{0}^{1}>0, p_{2}^{2}<1$. Уравнение $(4)$ для функции $w_{0}(z, u)$ является уравнением второго порядка

$$
\begin{aligned}
\left(\lambda_{2}\left(h_{2}^{2}(u)-1\right) z\right. & \left.+\lambda_{1} h_{2}^{1}(u)\right) \frac{d^{2} w_{0}(z, u)}{d z^{2}}+\left(\lambda_{2} h_{1}^{2}(u) z+\lambda_{1}\left(h_{1}^{1}(u)-1\right)\right) \frac{d w_{0}(z, u)}{d z} \\
& +\left(\lambda_{2} h_{0}^{2}(u) z+\lambda_{1} h_{0}^{1}(u)\right) w_{0}(z, u)=0 .
\end{aligned}
$$

Пусть функции $h_{\gamma_{1}}^{l}(u), \gamma_{1}=0,1,2, l=1,2$, аналитичны при всех $u$. Обозначим $\varphi_{0}(u)$ и $\varphi_{1}(u)$ корни квадратного уравнения $h_{2}(s, u)-s^{2}=0$. Функции $\varphi_{0}(u), \varphi_{1}(u)$ аналитичны в области $|u|<1$ за исключением конечного числа точек. Выполнив интегрирование в (5), получаем общее решение уравнения (7) в виде $\left(\varphi_{0}(u) \neq \varphi_{1}(u)\right)$

$$
w_{0}(z, u)=\sum_{n=1}^{2} C_{n}(u) \int_{K^{n}} e^{\left(z+d_{0}(u)\right) s}\left(s-\varphi_{0}(u)\right)^{\nu_{0}(u)-1}\left(s-\varphi_{1}(u)\right)^{\nu_{1}(u)-1} d s
$$

где

$$
d_{0}(u)=\frac{\lambda_{1}}{\lambda_{2}} \frac{h_{2}^{1}(u)}{h_{2}^{2}(u)-1}, \quad \nu_{l}(u)=\frac{\lambda_{1}}{\lambda_{2}} \frac{h_{1}\left(\varphi_{l}(u), u\right)-\varphi_{l}(u)}{h_{2}^{\prime}\left(\varphi_{l}(u), u\right)-2 \varphi_{l}(u)}, \quad l=0,1,
$$

$C_{1}(u), C_{2}(u)$ - произвольные функции (здесь использовано обозначение $h_{2}^{\prime}(s, u)=$ $\left.\partial h_{2}(s, u) / \partial s\right)$. Кривые $K^{1}, K^{2}$ удовлетворяют условию (6); интегралы по ним являются линейно независимыми функциями переменной $z$. Отметим, что функции $\nu_{0}(u)$ и $\nu_{1}(u)$ определены так же, как параметры $\alpha$ и $\beta$ в работе [7].

Функция $d_{0}(u)$ аналитична при $|u|<1$. Аналитичность функций $\nu_{0}(u), \nu_{1}(u)$ в области $|u|<1$ за исключением конечного числа точек следует из равенства $h_{2}^{\prime}\left(\varphi_{l}(u), u\right)-2 \varphi_{l}(u)=(-1)^{l}\left(h_{2}^{2}(u)-1\right)\left(\varphi_{0}(u)-\varphi_{1}(u)\right), l=0,1$.

Выбор кривых интегрирования в выражении (8) связан с рассмотрением точек ветвления $\varphi_{0}(u)$ и $\varphi_{1}(u)$ подынтегральных функций. Если кривая $K^{1}$ имеет вид двойной замкнутой петли, обходящей точки $\varphi_{0}(u)$ и $\varphi_{1}(u)$ сначала в положительном, а потом в отрицательном направлениях (ср. $[9$, гл. $6, \S 11$, интегральное представление (1)]), то интеграл (8) дает функцию, аналитическую в области $|u|<1$ за исключением конечного числа точек, В которых $\varphi_{0}(u)=\varphi_{1}(u)$. Значения функции $w_{0}(z, u)$ в указанных точках определяются по непрерывности.

Далее используется другое интегральное представление для $w_{0}(z, u)$, справедливое в окрестности точки $u=1$. Так как $h_{2}(1,1)=1$, положим $\varphi_{0}(1)=1$. Из условий $p_{2}^{2}<1$ и $\varphi_{0}(1)=1$ следует $-1 \leqslant \varphi_{1}(1) \leqslant 0$. Отсюда, учитывая, что $h_{1}(1,1)=1$ и $p_{0}^{1}>0$, получаем $\nu_{0}(1)=0, \nu_{1}(1)>0$. Следовательно, в силу непрерывности функции $\nu_{1}(u)$ в точке $u=1$, неравенство $\operatorname{Re} \nu_{1}(u)>0$ справедливо в области $|u-1|<\varepsilon$ при некотором $\varepsilon>0$. При $\operatorname{Re} \nu_{1}(u)>0$ в качестве кривой $K^{1}$ можно взять петлю, которая начинается и заканчивается в точке $\varphi_{1}(u)$ и обходит в положительном направлении точку $\varphi_{0}(u)$ (ср. $\left[9\right.$, гл. $6, \S 11$, интегральное представление (3)]). Кривая $K^{2}$ берется 
в виде бесконечной петли, проходящей вдоль разреза по лучу $\arg \left(s-\varphi_{1}(u)\right)=\theta$, $\operatorname{Re}\left(\left(z+d_{0}(u)\right) e^{i \theta}\right)<0$ и обходящей точку $\varphi_{1}(u)$ в положительном направлении (ср. $[9$, гл. $6, \S 11$, интегральное представление (9)]). Поскольку функция, определяемая интегралом по кривой $K^{2}$, не является аналитической при $z=-d_{0}(u)$, полагаем $C_{2}(u) \equiv 0$. Функция $C_{1}(u)$ определяется из условия $w_{0}(0, u) \equiv 1$. После замены переменной $s=\varphi_{0}(u)+\left(\varphi_{1}(u)-\varphi_{0}(u)\right) v$ в (8) приходим к представлению вида (далее $\left.d(u)=d_{0}(u)\left(\varphi_{1}(u)-\varphi_{0}(u)\right)\right)$

где

$$
w_{0}(z, u)=\frac{1}{A(u)} \int_{1}^{(0+)} e^{z\left(\varphi_{0}(u)+\left(\varphi_{1}(u)-\varphi_{0}(u)\right) v\right)+d(u) v} v^{\nu_{0}(u)-1}(1-v)^{\nu_{1}(u)-1} d v,
$$

$$
A(u)=\int_{1}^{(0+)} e^{d(u) v} v^{\nu_{0}(u)-1}(1-v)^{\nu_{1}(u)-1} d v, \quad|u-1|<\varepsilon .
$$

Теорема 2. Пусть для ветвящегося прочесса $h_{1}(s, u)=h_{0}^{1}(u)+h_{1}^{1}(u) s+$ $h_{2}^{1}(u) s^{2}, h_{2}(s, u)=h_{0}^{2}(u)+h_{1}^{2}(u) s+h_{2}^{2}(u) s^{2} u p_{0}^{1}>0, p_{2}^{2}<1$. Производящая функиия финального распределения при $|u-1|<\varepsilon$ представима в виде

$$
f_{\alpha_{1}}(u)=\frac{1}{A(u)} \int_{1}^{(0+)} e^{d(u) v}(1-v)^{\nu_{1}(u)-1} \frac{\left(\varphi_{0}(u)+\left(\varphi_{1}(u)-\varphi_{0}(u)\right) v\right)^{\alpha_{1}}}{v^{1-\nu_{0}(u)}} d v .
$$

Формула (11) следует из интегрального представления (9) и определения (2).

Далее полагаем, что контур интегрирования в (10) и (11) состоит из отрезка, ориентированного от $v=1$ до $v=r, 0<r<1$, окружности $|v|=r$ и отрезка от $v=r$ до $v=1$. Поскольку подынтегральные функции при $u=1$ однозначны в области $|v| \leqslant 1$, интегрирование по отрезку $[r, 1]$ в противоположных направлениях дает нуль. Вычисляя интегралы по окружности $|v|=r$ через вычеты в полюсе первого порядка $v=0$, находим $A(1)=2 \pi i$ и $f_{\alpha_{1}}(1)=1$. В частности, для вероятности вырождения получаем $q_{\alpha_{1} 0}=1, \alpha_{1} \in \mathbf{N}$.

3 а м е ч а н и е 1. Уравнение (7) сводится к вырожденному гипергеометрическому уравнению (см. [10, ч. 3, гл. 2, уравнения $2.113,2.145],[8$, гл. 16, пример 5]). Экспоненциальная производящая функция представляется в виде

где

$$
w_{0}(z, u)=e^{z \varphi_{0}(u)} \frac{\Phi\left(\nu_{0}(u), \nu_{0}(u)+\nu_{1}(u) ; z\left(\varphi_{1}(u)-\varphi_{0}(u)\right)+d(u)\right)}{\Phi\left(\nu_{0}(u), \nu_{0}(u)+\nu_{1}(u) ; d(u)\right)},
$$

$$
\Phi(a, b ; z)=1+\sum_{n=1}^{\infty} \frac{a(a+1) \cdots(a+n-1)}{b(b+1) \cdots(b+n-1)} \frac{z^{n}}{n !}, \quad b \neq 0,-1,-2, \ldots,
$$

- вырожденная гипергеометрическая функция [9]. Представление (12) имеет место при $|u| \leqslant 1$ за исключением конечного числа точек.

5. Математическое ожидание финального распределения. Случай $h_{1}(s, u)=h_{0}^{1}(u)+h_{1}^{1}(u) s+h_{2}^{1}(u) s^{2}, h_{2}(s, u)=h_{0}^{2}(u)+h_{1}^{2}(u) s+h_{2}^{2}(u) s^{2}, p_{0}^{1}>0, p_{2}^{2}<1$. Вычислим математическое ожидание $m_{\alpha_{1}}=f_{\alpha_{1}}^{\prime}(1)$. Дифференцируя дробь (11), имеем $\left(\alpha_{1}>0\right)$

$$
\begin{aligned}
m_{\alpha_{1}}= & \left.\frac{1}{2 \pi i} \int_{1}^{(0+)} \frac{d}{d u}\left(e^{d(u) v}(1-v)^{\nu_{1}(u)-1} \frac{\left(\varphi_{0}(u)+\left(\varphi_{1}(u)-\varphi_{0}(u)\right) v\right)^{\alpha_{1}}-1}{v^{1-\nu_{0}(u)}}\right)\right|_{u=1} d v \\
= & \frac{d^{\prime}(1)}{2 \pi i} \int_{1}^{(0+)} e^{d(1) v}(1-v)^{\nu_{1}(1)-1}\left(\left(1+\left(\varphi_{1}(1)-1\right) v\right)^{\alpha_{1}}-1\right) d v \\
& -\frac{\nu_{1}^{\prime}(1)}{2 \pi i} \int_{1}^{(0+)} e^{d(1) v}(1-v)^{\nu_{1}(1)-1} \ln (1-v) \cdot \frac{\left(1+\left(\varphi_{1}(1)-1\right) v\right)^{\alpha_{1}}-1}{v} d v \\
& +\frac{\nu_{0}^{\prime}(1)}{2 \pi i} \int_{1}^{(0+)} e^{d(1) v}(1-v)^{\nu_{1}(1)-1} \ln v \cdot \frac{\left(1+\left(\varphi_{1}(1)-1\right) v\right)^{\alpha_{1}}-1}{v} d v \\
& +\frac{\alpha_{1}}{2 \pi i} \int_{1}^{(0+)} e^{d(1) v}(1-v)^{\nu_{1}(1)-1}\left(\varphi_{0}^{\prime}(1)+\left(\varphi_{1}^{\prime}(1)-\varphi_{0}^{\prime}(1)\right) v\right) \\
& \times \frac{\left(1+\left(\varphi_{1}(1)-1\right) v\right)^{\alpha_{1}-1}}{v} d v .
\end{aligned}
$$


Интеграл (14) равен нулю, так как подынтегральная функция не имеет особых точек при $|v|<1$. Вычисляя вычеты в точке $v=0$, получаем, что интеграл (15) равен нулю, а интеграл (16) равен $\varphi_{0}^{\prime}(1) \alpha_{1}$. Таким образом, для математического ожидания имеем

$$
m_{\alpha_{1}}=\varphi_{0}^{\prime}(1) \alpha_{1}-\frac{\nu_{0}^{\prime}(1)}{2 \pi i} \int_{1}^{(0+)} e^{d(1) v}(1-v)^{\nu_{1}(1)-1} \ln v \cdot \frac{1-\left(1-\left(1-\varphi_{1}(1)\right) v\right)^{\alpha_{1}}}{v} d v .
$$

Преобразуем выражение под интегралом (17), используя сумму геометрической прогрессии $1-\left(1-\left(1-\varphi_{1}(1)\right) v\right)^{\alpha_{1}}=\left(1-\varphi_{1}(1)\right) v \sum_{k=0}^{\alpha_{1}-1}\left(1-\left(1-\varphi_{1}(1)\right) v\right)^{k}$, а также разложения бинома и экспоненты по степеням $1-v$. Имеем

$$
\begin{aligned}
(1- & \left.\varphi_{1}(1)\right) \int_{1}^{(0+)} e^{d(1) v}(1-v)^{\nu_{1}(1)-1} \ln v \cdot \sum_{k=0}^{\alpha_{1}-1}\left(\left(1-\varphi_{1}(1)\right)(1-v)+\varphi_{1}(1)\right)^{k} d v \\
= & e^{d(1)} \sum_{k=0}^{\alpha_{1}-1} \sum_{l=0}^{k} C_{k}^{l}\left(1-\varphi_{1}(1)\right)^{l+1} \varphi_{1}^{k-l}(1) \\
& \times \sum_{m=0}^{\infty} \frac{(-d(1))^{m}}{m !} \int_{1}^{(0+)}(1-v)^{\nu_{1}(1)+l+m-1} \ln v d v .
\end{aligned}
$$

К контурному интегралу применяем интегрирование по частям и после вычисления вычета в точке $v=0$ переходим к интегралу по действительной полуоси:

$$
\begin{aligned}
\frac{1}{2 \pi i} \int_{1}^{(0+)}(1-v)^{\nu_{1}(1)+l+m-1} \ln v d v & =\frac{1}{2 \pi i} \int_{1}^{(0+)} \frac{(1-v)^{\nu_{1}(1)+l+m}}{\left(\nu_{1}(1)+l+m\right) v} d v \\
& =\frac{1}{\nu_{1}(1)+l+m}=\int_{0}^{+\infty} e^{-\left(\nu_{1}(1)+l+m\right) v} d v
\end{aligned}
$$

После подстановки последнего интеграла в (18) и суммирования выражение (17) приобретает вид

$$
m_{\alpha_{1}}=\varphi_{0}^{\prime}(1) \alpha_{1}-\nu_{0}^{\prime}(1) \int_{0}^{+\infty} e^{d(1)\left(1-e^{-v}\right)-\nu_{1}(1) v} \frac{1-\left(\left(1-\varphi_{1}(1)\right) e^{-v}+\varphi_{1}(1)\right)^{\alpha_{1}}}{1-e^{-v}} d v
$$

Теорема 3. Пусть выполнены условия теоремы 2. Для математического ожидания финального распределения имеет место асимптотическая оценка

$$
m_{\alpha_{1}}=\varphi_{0}^{\prime}(1) \alpha_{1}-\nu_{0}^{\prime}(1) \ln \alpha_{1}+O(1), \quad \alpha_{1} \rightarrow \infty .
$$

Д ок а з а т е л ь с в о. Используя представление логарифма

$$
\ln x=\int_{0}^{+\infty} \frac{e^{-v}-e^{-x v}}{v} d v, \quad x>0,
$$

$[9$, гл. $1, \S 7$, формула (18)], разобьем интеграл в выражении (19) на сумму интегралов. Тогда

$$
\begin{aligned}
& \int_{0}^{+\infty} e^{d(1)\left(1-e^{-v}\right)-\nu_{1}(1) v} \frac{1-\left(\left(1-\varphi_{1}(1)\right) e^{-v}+\varphi_{1}(1)\right)^{\alpha_{1}}}{1-e^{-v}} d v \\
& =\ln \left(\left(1-\varphi_{1}(1)\right) \alpha_{1}+\nu_{1}(1)\right)+\int_{0}^{+\infty}\left(\frac{e^{d(1)\left(1-e^{-v}\right)-\nu_{1}(1) v}}{1-e^{-v}}-\frac{e^{-v}}{v}\right) d v \\
& \quad+\int_{0}^{+\infty}\left(\frac{1}{v}-\frac{1}{1-e^{-v}}\right) e^{d(1)\left(1-e^{-v}\right)-\nu_{1}(1) v}\left(\left(1-\varphi_{1}(1)\right) e^{-v}+\varphi_{1}(1)\right)^{\alpha_{1}} d v \\
& +\int_{0}^{+\infty} \frac{1-e^{d(1)\left(1-e^{-v}\right)}}{v} e^{-\left(\left(1-\varphi_{1}(1)\right) \alpha_{1}+\nu_{1}(1)\right) v} d v \\
& +\int_{0}^{\ln \left(1-1 / \varphi_{1}(1)\right)} \frac{e^{-\left(1-\varphi_{1}(1)\right) \alpha_{1} v}-\left(\left(1-\varphi_{1}(1)\right) e^{-v}+\varphi_{1}(1)\right)^{\alpha_{1}}}{v} \\
& \times e^{d(1)\left(1-e^{-v}\right)-\nu_{1}(1) v} d v
\end{aligned}
$$




$$
\begin{gathered}
+\int_{\ln \left(1-1 / \varphi_{1}(1)\right)}^{+\infty} \frac{e^{-\left(1-\varphi_{1}(1)\right) \alpha_{1} v}-\left(\left(1-\varphi_{1}(1)\right) e^{-v}+\varphi_{1}(1)\right)^{\alpha_{1}}}{v} \\
\times e^{d(1)\left(1-e^{-v}\right)-\nu_{1}(1) v} d v .
\end{gathered}
$$

В случае $\varphi_{1}(1)=0$ слагаемые (24) и (25) отсутствуют. В интегралах (21)-(24) подынтегральные функции ограничены при $v \rightarrow 0+$, поэтому интегралы сходятся. Интегралы (22), (23) и (25) стремятся к нулю при $\alpha_{1} \rightarrow \infty$. Для оценки интеграла (24) воспользуемся неравенством $1-x^{\alpha_{1}} \leqslant \alpha_{1}(1-x)$ при $0<x<1$ и ограниченностью функции

$$
y(v)=\frac{e^{-\left(1-\varphi_{1}(1)\right) v}-\left(1-\varphi_{1}(1)\right) e^{-v}-\varphi_{1}(1)}{v e^{-\left(1-\varphi_{1}(1)\right) v}}, \quad 0<v<\ln \left(1-\frac{1}{\varphi_{1}(1)}\right)
$$

(так как $\lim _{v \rightarrow 0} y(v)=0$ ). Имеем для $(24)$

$$
\begin{aligned}
& \int_{0}^{\ln \left(1-1 / \varphi_{1}(1)\right)}\left(1-\left(\frac{\left(1-\varphi_{1}(1)\right) e^{-v}+\varphi_{1}(1)}{e^{-\left(1-\varphi_{1}(1)\right) v}}\right)^{\alpha_{1}}\right) \frac{e^{d(1)\left(1-e^{-v}\right)-\nu_{1}(1) v-\left(1-\varphi_{1}(1)\right) \alpha_{1} v}}{v} d v \\
& \quad \leqslant \alpha_{1} \int_{0}^{\ln \left(1-1 / \varphi_{1}(1)\right)} y(v) e^{d(1)\left(1-e^{-v}\right)-\nu_{1}(1) v-\left(1-\varphi_{1}(1)\right) \alpha_{1} v} d v \\
& \leqslant \alpha_{1} e^{d(1)} \sup y(v) \int_{0}^{\ln \left(1-1 / \varphi_{1}(1)\right)} e^{-\left(1-\varphi_{1}(1)\right) \alpha_{1} v} d v \leqslant \frac{e^{d(1)}}{1-\varphi_{1}(1)} \sup y(v) .
\end{aligned}
$$

Из ограниченности значений интегралов (21)-(25) с учетом равенства $\ln ((1-$ $\left.\left.\varphi_{1}(1)\right) \alpha_{1}+\nu_{1}(1)\right)=\ln \alpha_{1}+O(1), \alpha_{1} \rightarrow \infty$, получаем оценку (20). Теорема доказана.

При $h_{2}(s, u)=p_{0}^{2}+p_{1}^{2} s$ (парные взаимодействия не дают частиц типа $T_{2}$ ) имеем $\varphi_{0}(u) \equiv 1, \varphi_{1}(u) \equiv-p_{0}^{2}$ и $m_{\alpha_{1}}=\left(\lambda_{1} / \lambda_{2}\right) a_{2}^{1} \ln \alpha_{1} /\left(1+p_{0}^{2}\right)+O(1), \alpha_{1} \rightarrow \infty$, где $a_{2}^{1}=\left.\left(\partial h_{1}(s, u) / \partial u\right)\right|_{s=1, u=1}$ - среднее число частиц типа $T_{2}$, образующихся при превращении частицы типа $T_{1}[12]$.

Возможность исследования асимптотики дисперсии финального распределения при $\alpha_{1} \rightarrow \infty$, исходя из представления (11), остается неясной.

6. Предельная теорема для финального распределения. Случай $h_{1}(s, u)=h_{0}^{1}(u)+h_{1}^{1}(u) s, h_{2}(s, u)=h_{1}^{2}(u) s+h_{2}^{2}(u) s^{2}, p_{0}^{1}>0, p_{2}^{2}<1$. B pacсматриваемом случае $\varphi_{0}(u)=h_{1}^{2}(u) /\left(1-h_{2}^{2}(u)\right), \varphi_{1}(u) \equiv 0$ и $d(u) \equiv 0$. Учитывая равенство $\Phi(a, b ; z)=e^{z} \Phi(b-a, b ;-z)[9$, гл. $6, \S 3]$, из формулы (12) имеем $w_{0}(z, u)=\Phi\left(\nu_{1}(u), \nu_{0}(u)+\nu_{1}(u) ; \varphi_{0}(u) z\right)$. Используя (13), приравнивая коэффициенты при одинаковых степенях $z$ в ряде для $w_{0}(z, u)$ и в ряде $(2)$, получаем производящую функцию финального распределения $\left(\alpha_{1}>0\right)$

$$
f_{\alpha_{1}}(u)=\varphi_{0}^{\alpha_{1}}(u) \frac{\nu_{1}(u) \cdots\left(\nu_{1}(u)+\alpha_{1}-1\right)}{\left(\nu_{0}(u)+\nu_{1}(u)\right) \cdots\left(\nu_{0}(u)+\nu_{1}(u)+\alpha_{1}-1\right)}, \quad|u| \leqslant 1 .
$$

Учитывая свойство гамма-функции $\Gamma\left(z+\alpha_{1}\right)=z(z+1) \cdots\left(z+\alpha_{1}-1\right) \Gamma(z)$, приходим к выражению

$$
f_{\alpha_{1}}(u)=\varphi_{0}^{\alpha_{1}}(u) \frac{\Gamma\left(\nu_{1}(u)+\alpha_{1}\right) \Gamma\left(\nu_{0}(u)+\nu_{1}(u)\right)}{\Gamma\left(\nu_{0}(u)+\nu_{1}(u)+\alpha_{1}\right) \Gamma\left(\nu_{1}(u)\right)} .
$$

Используя выражения $\nu_{1}(u)=\left(\lambda_{1} / \lambda_{2}\right) h_{0}^{1}(u) / h_{1}^{2}(u), \nu_{0}(u)+\nu_{1}(u)=\left(\lambda_{1} / \lambda_{2}\right)(1-$ $\left.h_{1}^{1}(u)\right) /\left(1-h_{2}^{2}(u)\right)$, можно доказать аналитичность функции $(26)$ в области $|u|<1+\varepsilon$ при некотором $\varepsilon>0$. Вычислим математическое ожидание $m_{\alpha_{1}}=f_{\alpha_{1}}^{\prime}(1)$ и дисперсию $\sigma_{\alpha_{1}}^{2}=f_{\alpha_{1}}^{\prime \prime}(1)+f_{\alpha_{1}}^{\prime}(1)-\left(f_{\alpha_{1}}^{\prime}(1)\right)^{2}$. При дифференцировании (27) воспользуемся функцией $\psi(z)=d \ln \Gamma(z) / d z[9$, гл. $1, \S 7],[8$, гл. 12]. Получаем выражения

$$
\begin{aligned}
m_{\alpha_{1}}= & \varphi_{0}^{\prime}(1) \alpha_{1}-\nu_{0}^{\prime}(1)\left(\psi\left(\nu_{1}(1)+\alpha_{1}\right)-\psi\left(\nu_{1}(1)\right)\right), \\
\sigma_{\alpha_{1}}^{2}= & \left(\varphi_{0}^{\prime \prime}(1)+\varphi_{0}^{\prime}(1)-\left(\varphi_{0}^{\prime}(1)\right)^{2}\right) \alpha_{1}-\left(\nu_{0}^{\prime \prime}(1)+\nu_{0}^{\prime}(1)\right)\left(\psi\left(\nu_{1}(1)+\alpha_{1}\right)-\psi\left(\nu_{1}(1)\right)\right) \\
& -\nu_{0}^{\prime}(1)\left(\nu_{0}^{\prime}(1)+2 \nu_{1}^{\prime}(1)\right)\left(\psi^{\prime}\left(\nu_{1}(1)+\alpha_{1}\right)-\psi^{\prime}\left(\nu_{1}(1)\right)\right) .
\end{aligned}
$$

Формула (28) следует также из (19) и интегрального представления для функции $\psi(z)[9$, гл. $1, \S 7$, формула (17)]. 
Теорема 4. Пусть для ветвящегося прочесса $h_{1}(s, u)=h_{0}^{1}(u)+h_{1}^{1}(u) s$, $h_{2}(s, u)=h_{1}^{2}(u) s+h_{2}^{2}(u) s^{2} u p_{0}^{1}>0, p_{2}^{2}<1$. Для математического ожидания $u$ дисперсии финального распределения имеют место асимптотические формуль

$$
\begin{aligned}
m_{\alpha_{1}} & \sim \varphi_{0}^{\prime}(1) \alpha_{1}-\nu_{0}^{\prime}(1) \ln \alpha_{1}+\nu_{0}^{\prime}(1) \psi\left(\nu_{1}(1)\right), \\
\sigma_{\alpha_{1}}^{2} & \sim\left(\varphi_{0}^{\prime \prime}(1)+\varphi_{0}^{\prime}(1)-\left(\varphi_{0}^{\prime}(1)\right)^{2}\right) \alpha_{1}-\left(\nu_{0}^{\prime \prime}(1)+\nu_{0}^{\prime}(1)\right) \ln \alpha_{1}, \quad \alpha_{1} \rightarrow \infty .
\end{aligned}
$$

Для вывода асимптотик (30) и (31) используются выражения $(28),(29)$ и асимптотические формулы $\psi(z) \sim \ln z, \psi^{\prime}(z) \sim 1 / z$ при $z \rightarrow \infty,|\arg z|<\pi[9$, гл. $1, \S 18]$.

Теорема 5. Пусть выполнень условия теоремь $4, \partial h_{1}(s, u) / \partial u \not \equiv 0$ или $\partial h_{2}(s, u) / \partial u \not \equiv 0$. При фиксированном $x \in(-\infty, \infty)$

$$
\lim _{\alpha_{1} \rightarrow \infty} \mathbf{P}\left\{\frac{\eta_{\alpha_{1}}-m_{\alpha_{1}}}{\sigma_{\alpha_{1}}}<x\right\}=\frac{1}{\sqrt{2 \pi}} \int_{-\infty}^{x} e^{-y^{2} / 2} d y
$$

Д ок а з а т е л ь с т в о. Покажем, что характеристическая функция $\hat{f}_{\alpha_{1}}(\tau)=$ $\mathbf{E} \exp \left(i \tau\left(\eta_{\alpha_{1}}-m_{\alpha_{1}}\right) / \sigma_{\alpha_{1}}\right)$ нормированной случайной величины $\left(\eta_{\alpha_{1}}-m_{\alpha_{1}}\right) / \sigma_{\alpha_{1}}$ стремится при $\alpha_{1} \rightarrow \infty$ к функции $\exp \left(-\tau^{2} / 2\right)$, являющейся характеристической функцией стандартного нормального распределения [3].

Введем функцию $y_{\alpha_{1}}(\tau)=\exp \left(i \tau / \sigma_{\alpha_{1}}\right)$. Тогда $\hat{f}_{\alpha_{1}}(\tau)=y_{\alpha_{1}}\left(-m_{\alpha_{1}} \tau\right) f_{\alpha_{1}}\left(y_{\alpha_{1}}(\tau)\right)$, и из (27) имеем

$$
\hat{f}_{\alpha_{1}}(\tau)=y_{\alpha_{1}}\left(-m_{\alpha_{1}} \tau\right) \varphi_{0}^{\alpha_{1}}\left(y_{\alpha_{1}}(\tau)\right) \frac{\Gamma\left(\nu_{1}\left(y_{\alpha_{1}}(\tau)\right)+\alpha_{1}\right) \Gamma\left(\nu_{0}\left(y_{\alpha_{1}}(\tau)\right)+\nu_{1}\left(y_{\alpha_{1}}(\tau)\right)\right)}{\Gamma\left(\nu_{0}\left(y_{\alpha_{1}}(\tau)\right)+\nu_{1}\left(y_{\alpha_{1}}(\tau)\right)+\alpha_{1}\right) \Gamma\left(\nu_{1}\left(y_{\alpha_{1}}(\tau)\right)\right)} .
$$

Используя разложение Тейлора $\ln \varphi_{0}\left(e^{v}\right)=\varphi_{0}^{\prime}(1) v+\left(\varphi_{0}^{\prime \prime}(1)+\varphi_{0}^{\prime}(1)-\left(\varphi_{0}^{\prime}(1)\right)^{2}\right) v^{2} / 2+$ $o\left(v^{2}\right), v \rightarrow 0$, получаем при $\alpha_{1} \rightarrow \infty$

$$
\varphi_{0}^{\alpha_{1}}\left(y_{\alpha_{1}}(\tau)\right)=\exp \left(i \tau \frac{\varphi_{0}^{\prime}(1) \alpha_{1}}{\sigma_{\alpha_{1}}}-\tau^{2} \frac{\left(\varphi_{0}^{\prime \prime}(1)+\varphi_{0}^{\prime}(1)-\left(\varphi_{0}^{\prime}(1)\right)^{2}\right) \alpha_{1}}{2 \sigma_{\alpha_{1}}^{2}}+o\left(\frac{\alpha_{1}}{\sigma_{\alpha_{1}}^{2}}\right)\right) .
$$

С помощью формулы Стирлинга $\Gamma(z) \sim \sqrt{2 \pi} \exp \left(\left(z-\frac{1}{2}\right) \ln z-z\right), z \rightarrow \infty$, $|\arg z|<\pi$, получаем

$$
\begin{aligned}
& \frac{\Gamma\left(\nu_{1}\left(y_{\alpha_{1}}(\tau)\right)+\alpha_{1}\right)}{\Gamma\left(\nu_{0}\left(y_{\alpha_{1}}(\tau)\right)+\nu_{1}\left(y_{\alpha_{1}}(\tau)\right)+\alpha_{1}\right)} \\
& \sim \exp \left(\left(\nu_{1}\left(y_{\alpha_{1}}(\tau)\right)+\alpha_{1}-\frac{1}{2}\right) \ln \left(1-\frac{\nu_{0}\left(y_{\alpha_{1}}(\tau)\right)}{\nu_{0}\left(y_{\alpha_{1}}(\tau)\right)+\nu_{1}\left(y_{\alpha_{1}}(\tau)\right)+\alpha_{1}}\right)\right. \\
& \left.\quad-\nu_{0}\left(y_{\alpha_{1}}(\tau)\right) \ln \left(\nu_{0}\left(y_{\alpha_{1}}(\tau)\right)+\nu_{1}\left(y_{\alpha_{1}}(\tau)\right)+\alpha_{1}\right)+\nu_{0}\left(y_{\alpha_{1}}(\tau)\right)\right) .
\end{aligned}
$$

Поскольку $\ln (1+z) \sim z, z \rightarrow 0$, то, учитывая, что $\nu_{0}\left(y_{\alpha_{1}}(\tau)\right) \rightarrow 0, \nu_{1}\left(y_{\alpha_{1}}(\tau)\right) \rightarrow \nu_{1}(1)$ при $\alpha_{1} \rightarrow \infty$, первое слагаемое под знаком экспоненты в выражении (34) стремится к нулю. Так как $\ln \left(\nu_{0}\left(y_{\alpha_{1}}(\tau)\right)+\nu_{1}\left(y_{\alpha_{1}}(\tau)\right)+\alpha_{1}\right) \sim \ln \alpha_{1}, \alpha_{1} \rightarrow \infty$, то имеем

$$
\frac{\Gamma\left(\nu_{1}\left(y_{\alpha_{1}}(\tau)\right)+\alpha_{1}\right)}{\Gamma\left(\nu_{0}\left(y_{\alpha_{1}}(\tau)\right)+\nu_{1}\left(y_{\alpha_{1}}(\tau)\right)+\alpha_{1}\right)} \sim \exp \left(-\nu_{0}\left(y_{\alpha_{1}}(\tau)\right) \ln \alpha_{1}\right)
$$

Применяя разложение Тейлора $\nu_{0}\left(e^{v}\right)=\nu_{0}^{\prime}(1) v+\left(\nu_{0}^{\prime \prime}(1)+\nu_{0}^{\prime}(1)\right) v^{2} / 2+o\left(v^{2}\right), v \rightarrow 0$, из (35) получаем при $\alpha_{1} \rightarrow \infty$

$$
\begin{aligned}
& \frac{\Gamma\left(\nu_{1}\left(y_{\alpha_{1}}(\tau)\right)+\alpha_{1}\right)}{\Gamma\left(\nu_{0}\left(y_{\alpha_{1}}(\tau)\right)+\nu_{1}\left(y_{\alpha_{1}}(\tau)\right)+\alpha_{1}\right)} \\
& \quad=\exp \left(-i \tau \frac{\nu_{0}^{\prime}(1) \ln \alpha_{1}}{\sigma_{\alpha_{1}}}+\tau^{2} \frac{\left(\nu_{0}^{\prime \prime}(1)+\nu_{0}^{\prime}(1)\right) \ln \alpha_{1}}{2 \sigma_{\alpha_{1}}^{2}}+o\left(\frac{\ln \alpha_{1}}{\sigma_{\alpha_{1}}^{2}}\right)\right) .
\end{aligned}
$$

Перемножая $y_{\alpha_{1}}\left(-m_{\alpha_{1}} \tau\right)$, выражения (33), (36) и учитывая, что

$$
\Gamma\left(\nu_{0}\left(y_{\alpha_{1}}(\tau)\right)+\nu_{1}\left(y_{\alpha_{1}}(\tau)\right)\right) \sim \Gamma\left(\nu_{1}\left(y_{\alpha_{1}}(\tau)\right)\right), \quad \alpha_{1} \rightarrow \infty,
$$


имеем при $h_{2}(s, u) \neq s$

$$
\begin{aligned}
\hat{f}_{\alpha_{1}}(\tau)=\exp ( & i \tau \frac{\varphi_{0}^{\prime}(1) \alpha_{1}-\nu_{0}^{\prime}(1) \ln \alpha_{1}-m_{\alpha_{1}}}{\sigma_{\alpha_{1}}} \\
& \left.-\tau^{2} \frac{\left(\varphi_{0}^{\prime \prime}(1)+\varphi_{0}^{\prime}(1)-\left(\varphi_{0}^{\prime}(1)\right)^{2}\right) \alpha_{1}-\left(\nu_{0}^{\prime \prime}(1)+\nu_{0}^{\prime}(1)\right) \ln \alpha_{1}}{2 \sigma_{\alpha_{1}}^{2}}+o\left(\frac{\alpha_{1}}{\sigma_{\alpha_{1}}^{2}}\right)\right) .
\end{aligned}
$$

При $h_{2}(s, u)=s$ имеем $\varphi_{0}(u) \equiv 1$, и остаточный член в последней формуле равен $o\left(\ln \alpha_{1} / \sigma_{\alpha_{1}}^{2}\right)$. Из оценки (30) следует, что первое слагаемое под знаком экспоненты в выражении (37) стремится к нулю. Применяя ко второму слагаемому и остаточному члену асимптотику (31), получаем

$$
\lim _{\alpha_{1} \rightarrow \infty} \hat{f}_{\alpha_{1}}(\tau)=\exp \left(-\frac{\tau^{2}}{2}\right) .
$$

Теорема доказана.

3 а м е ч а н и е 2. Производящая функция финальных вероятностей (26) записывается в виде произведения

$$
f_{\alpha_{1}}(u)=g_{1}(u) \cdots g_{\alpha_{1}}(u)
$$

где $g_{n}(u)=\varphi_{0}(u)\left(\nu_{1}(u)+n-1\right) /\left(\nu_{0}(u)+\nu_{1}(u)+n-1\right), n=1, \ldots, \alpha_{1},-$ вероятностные производяшие функции. Применение теоремы Ляпунова $[3, \S 40]$ к сумме $\eta_{\alpha_{1}}=\eta^{1}+$ $\cdots+\eta^{\alpha_{1}}$ независимых случайных величин $\eta^{n}$ с производящими функциями $g_{n}(u) \mathrm{c}$ целью вывода соотношения (32) требует проверки условия указанной теоремы.

\section{СПИСОК ЛИТЕРАТУРЫ}

1. Баруча-Рид А. Т. Элементы теории марковских процессов и их приложения. М.: Наука, 1969, 512 с.

2. Ван Кампен Н.Г. Стохастические процессы в физике и химии. М.: Высшая школа, 1990, $376 \mathrm{c.}$

3. Гнеденко Б. В. Курс теории вероятностей. М.: УРСС, 2001, 318 с.

4. Севастьянов Б. А. Ветвящиеся процессы. М.: Наука, 1971, 436 с.

5. Севастьянов Б.А., Калинкин А.В. Ветвяшиеся случайные процессы с взаимодействием частиц. - Докл. АН СССР, 1982, т. 264, № 2, с. 306-308.

6. Калинкин A.В. Финальные вероятности для ветвящегося случайного процесса с взаимодействием частиц. - Докл. АН СССР, 1983, т. 269, № 6, с. 1309-1312.

7. Калинкин A.B. О вероятности вырождения ветвяшегося процесса с двумя комплексами взаимодействия частиц. - Теория вероятн. и ее примен., 2001, т. 46, B. 2, с. 376-381.

8. Уиттекер Э.Т., Ватсон Дж. Н. Курс современного анализа. Ч. 2: Трансцендентные функции. М.: Физматлит, 1963, 516 с.

9. Бейтмен $Г$., Эрдейи А. Высшие трансцендентные функции. Гипергеометрическая функция. Функция Лежандра. М.: Наука, 1973, 294 с.

10. Камке Э. Справочник по обыкновенным дифференциальным уравнениям. М.: Наука, 1971, $576 \mathrm{c.}$

11. Ланге А.М. Стационарное распределение в открытой стохастической системе с парным взаимодействием частиц. - Вестн. МГТУ им. Н.Э. Баумана, сер. естеств. науки, 2005, в. 1 (16), с. 3-22.

12. Ланге А.М. Асимптотические свойства финальных вероятностей одного ветвящегося процесса с взаимодействием частиц. - Обозр. прикл. и промышл. матем., 2005 , т. 12 , в. 3 , с. $669-770$. 